\title{
Acoustic multipath arrivals in the horizontal plane due to approaching nonlinear internal waves
}

\author{
Mohsen Badiey ${ }^{\text {a) }}$ \\ Physical Ocean Science and Engineering, University of Delaware, Newark, Delaware 19716 \\ badiey@udel.edu \\ Boris G. Katsnelson \\ Department of Physics, Voronezh State University, Voronezh, Russia 394006 \\ katz@phys.vsu.ru \\ Ying-Tsong Lin and James F. Lynch \\ Department of Applied Ocean Physics and Engineering, Woods Hole Oceanographic Institution, \\ Woods Hole, Massachusetts 02543 \\ ytlin@whoi.edu,jlynch@whoi.edu
}

\begin{abstract}
Simultaneous measurements of acoustic wave transmissions and a nonlinear internal wave packet approaching an along-shelf acoustic path during the Shallow Water 2006 experiment are reported. The incoming internal wave packet acts as a moving frontal layer reflecting (or refracting) sound in the horizontal plane. Received acoustic signals are filtered into acoustic normal mode arrivals. It is shown that a horizontal multipath interference is produced. This has previously been called a horizontal Lloyd's mirror. The interference between the direct path and the refracted path depends on the mode number and frequency of the acoustic signal. A mechanism for the multipath interference is shown. Preliminary modeling results of this dynamic interaction using vertical modes and horizontal parabolic equation models are in good agreement with the observed data.
\end{abstract}

(C) 2011 Acoustical Society of America

PACS numbers: 43.30.Re, 43.30.Es, 43.30.Dr, 43.30.Bp [GD]

Date Received: January 10, 2011 Date Accepted: January 18, 2011

\section{Introduction}

Experimental observation of a new three-dimensional, oceanographically induced effect is reported here. This effect appears to be due to the creation of a new horizontal multipath arrival when the nonlinear internal wave (NIW) front first approaches an acoustic track. The interference between the direct path arrival and the refracted path arrival caused by the interaction between acoustic waves and NIW front can create a "horizontal Lloyd's mirror" effect, as predicted in Ref. 1. Due to the motion of the NIW packet, the phenomena we are reporting here is inherently non-stationary. The magnitude of the acoustic intensity fluctuations is less than that of focusing/defocusing effects previously reported, ${ }^{2-4}$ making it a harder phenomenon to observe. Using simplified input parameters of the IWs measured during the experiment, vertical modes with the horizontal parabolic equation model are used to verify the proposed mechanism explaining this phenomenon.

\section{Experimental observation}

During the summer of 2006, a large multi-institutional, shallow water acoustics experiment, SW06, ${ }^{5}$ was conducted on the Mid-Atlantic Bight continental shelf at a location about $160 \mathrm{~km}$ east of the New Jersey coast and about $80 \mathrm{~km}$ southwest of the Hudson

\footnotetext{
${ }^{\text {a) }}$ Author to whom correspondence should be addressed.
} 
Canyon. In addition to the thermistors deployed to register the water column temperature variability, shipboard radar was used to observe the surface expressions of the IWs as they were generated at the shelf break and traveled shoreward. About 50 IW events were recorded for about 3 weeks when our main research vessel for this study, $\mathrm{R} / \mathrm{V}$ Sharp, was present in the field. Here, we present one of these events where a very good simultaneous record of the IW and acoustic wave propagation is established. This phenomenon is similarly observed for several other IW events during the experiment, but we will just focus on one here. The detailed experimental configuration for this part of the experiment is provided in Refs. 4 and 5.

We focus our attention on the IW train during a source transmission period between the geotimes $T=21: 30$ and 21:37 Greenwich Mean Time (GMT) on August 17,2006 . This 7-min transmission is just prior to the focusing event that was reported ${ }^{5}$ during the following transmission periods. The surface radar impressions of this event were recorded by two ships, R/V Sharp and R/V Oceanus, and provided detailed information on the position of the front of the NIW as it travels. While this IW event was propagating from its generation point near the shelf break toward shore, it approached the acoustic track between the NRL300 Hz sound source and the Woods Hole Oceanographic Institution (WHOI) array system, which has both vertical and horizontal line arrays (see Fig. 1 in Ref. 4). The NRL300 sound source was about $20 \mathrm{~km}$ to the north of the WHOI vertical line array (VLA). The approximate horizontal distance at $T=21: 30$ GMT from the source to the front of IW (the edge of the packet) is about 2 $\mathrm{km}$, and from the front to the WHOI VLA is about $400-500 \mathrm{~m}$; the corresponding angle between wave front and acoustic track is about $4^{\circ}$ to $5^{\circ}$. The crest of the first wave in the NIW packet reached the WHOI VLA at about 21:42 GMT, observed

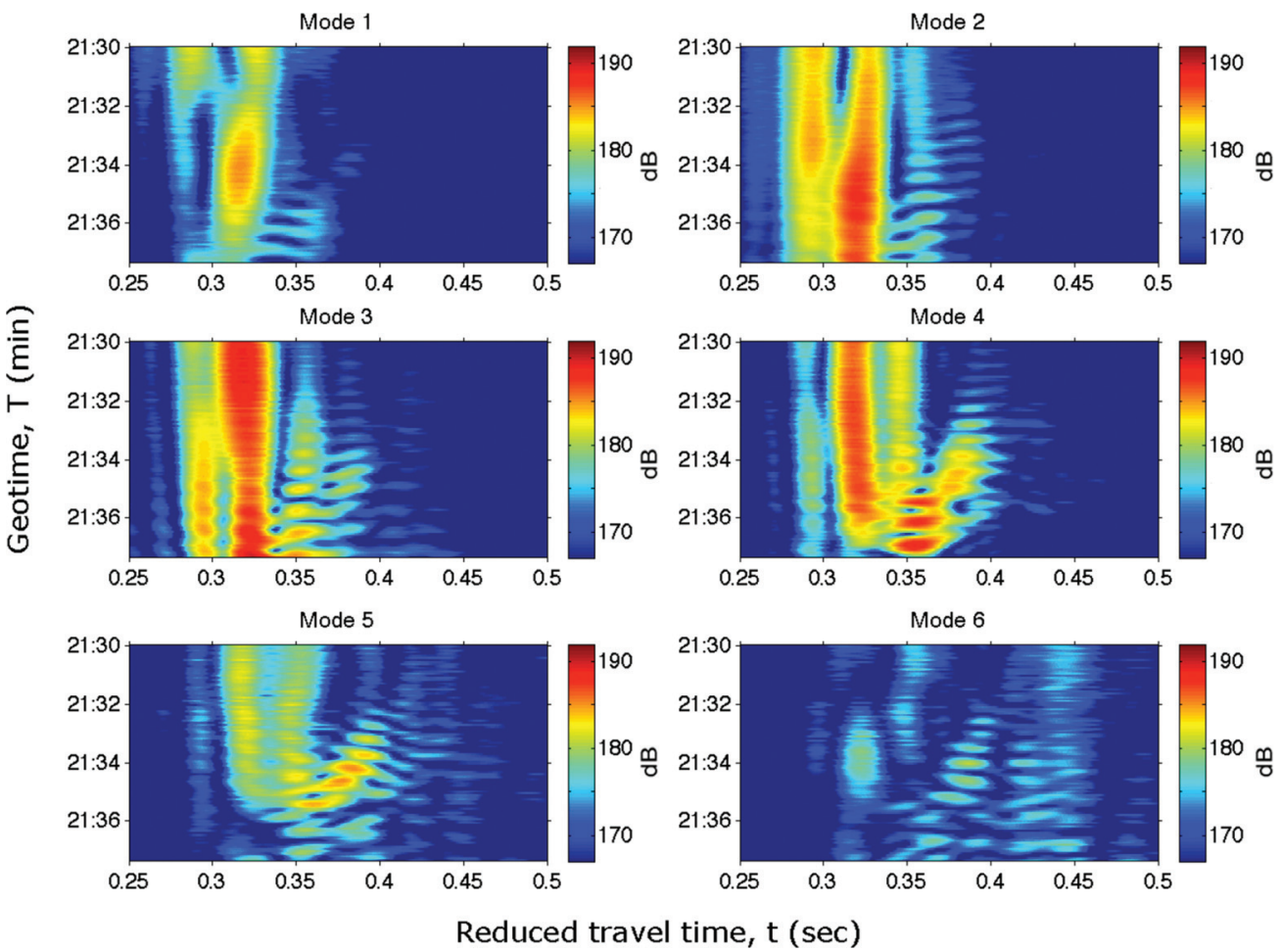

Fig. 1. Arrival of modes 1-6 filtered from the NRL300 sound signal received on the WHOI VLA array from geotime $T=21: 30-21: 37$ GMT on August 17, 2006. The earlier arrivals of modes 2 and 4 at about $t=0.325 \mathrm{~s}$ are cross talk from mode 3 . This imperfection of mode filtering is due to vertical array tilt, limited number of receivers, and limited aperture. 
from the temperature data on the VLA. From the shipboard radar images and the water column property profiles measured at three locations (i.e., at the source, at VLA, and at mid-point), a slight curvature on the wave front is observed. However, the modeling work in this paper assumes the front to be straight, and the curved front calculation with more detailed along-front variability of the NIW field is for future work.

Turning to the sources and receiver characteristics, a 16-channel VLA spanned the water column from $13.5 \mathrm{~m}$ depth to the bottom $(80 \mathrm{~m})$. The acoustic source transmitted a linearly swept chirp signal from 270 to $330 \mathrm{~Hz}$ in exactly $2.048 \mathrm{~s}$ followed by a 2.048-s long silence for 110 such periods (a total 7.5-min long transmission) at every hour and half hour. Each chirp was tapered with a $0.2048 \mathrm{~s}$ amplitude taper $(10 \%$ cosine taper) at the beginning and the end to allow gradually ramping on and off. The vertical modal content of the received signals on the WHOI VLA is examined by performing broadband mode filtering. The vertical mode functions used in the filter are calculated by the KRAKEN program using in situ water column sound speed profiles at the VLA location. The bottom model used for the normal mode calculation is from a previous study of the bottom geoacoustic properties. ${ }^{6}$

The observation addressed in this paper is the arrival of refracted path energy (here called secondary path) in the horizontal plane in addition to the direct path energy (primary path) in the received signal. Except for mode 6, which is very weak compared to the rest of the modes, both components of these energies exist in various levels of intensity in each mode. ${ }^{7}$ However, the ratio of the energies between the primary and secondary paths varies from mode to mode with the secondary path always smaller than the primary path energy. Since the goal here is only to establish the existence of the primary and secondary horizontal modal ray paths, and not the intermodal energy partitioning, we focus our attention on the mode that shows an almost equal energy ratio between these two, which is mode 4 . This mode most clearly shows the distinct multipath arrivals of the direct and the refracted paths in horizontal plane. Figure 1 shows the arrival of the first six modes obtained from data during the 7-min signal transmission from geotime $T=21: 30-21: 37$ GMT on August 17, 2006. It is noticed that most of the signal's energy is distributed among modes $2-4$, with smaller amplitude in modes 1 and 5 . It is noticed that modes 5 and 6 are very weak and much more dispersive than the other modes and mode 6 is almost non-existent.

The signal spread for each arriving mode in geotime starts with a primary arrival centered on $t=0.3 \mathrm{~s}$ during the $7 \mathrm{~min}$ transmission. For higher order modes, there is then a secondary arrival between $t=0.3$ and $t=0.4 \mathrm{~s}$ (for modes 5 and 6 , the spread is for $t=0.45 \mathrm{~s}$ ). The partitioning of energy during the 7 min geotime between the primary and the secondary arrivals varies for different modes. This is due to the transfer of energy between these two arrivals during the non-stationary behavior of the waveguide as the IW is approaching the acoustic track. Besides the arrival of the secondary modal energy, there are several higher order effects like the periodicity of the intensity fluctuations and some energy transfer among modes that occurs during this time. To address these finer points, more detailed modeling and 3D environmental reconstruction are required. To show the point of this paper, i.e., the appearance and existence of the secondary modal arrival, we choose one of the most prominent modes (such as mode 4) for discussion.

If we look at mode 4 in more detail, we see that at the beginning of the transmission, i.e., from $T=21: 30-21: 32$ GMT, the primary part of the signal's energy arrives between $t=0.257-0.36 \mathrm{~s}$. This energy continues to arrive throughout the entire $7 \mathrm{~min}$. Shortly after the beginning of transmission, a secondary ray appears arriving around $t=0.4 \mathrm{~s}$ and $T=21: 32$ GMT. As the NIW approaches the acoustic track, this arrival starts to become stronger and its amplitude grows with increasing geotime. From $T=21: 35$ GMT and beyond, it overlaps with the direct (primary) arrival path and contributes to the total received signal amplitude causing it to increase (see Fig. 1, mode 4). At $T=21: 37$, GMT transmissions stopped on the source's duty cycle schedule. 
Under cursory inspection, the appearance of the secondary arrival modal energy in Fig. 1 might be construed as signal processing error or as the result of mode coupling from the adjacent modes. Both these possibilities are addressed in the discussion section to follow. However, what we believe to be the correct mechanism for the arrival of the refracted signal is explained using the theory of horizontal rays and vertical modes. To show this, vertical mode and horizontal parabolic equation modeling is used with a simplified NIW input.

\section{Analysis}

In this section, we present a theoretical and computational explanation for the modal arrival patterns (Fig. 1; see mode 4). A single modal arrival for mode 4 is initially seen, but then one also sees a second arrival for this mode starting at approximately $T=21: 32$ GMT. This secondary arrival is the result of a horizontal total internal reflection/refraction phenomenon. There is a distinct critical angle regime for the reflections of each mode from an IW front, and this arrival is only seen when the critical angle criterion is satisfied.

A more precise interpretation can be provided using the theory of vertical modes and horizontal rays. ${ }^{2}$ Let us suppose that the plane wave front of an IW train is directed along the $X$-axis shown in Fig. 2(a). The internal solitary wave propagates in the $Y$-direction and is described by its surface of constant density $\zeta(\vec{r}, z, T)$ as a function of the spatial and temporal coordinates $\vec{r}=(x, y)$ in the horizontal plane. Here, $z$ is the depth and $T$ is the geotime. The sound field amplitude $P(\vec{r}, z, t)$ from a point source with spectrum $S(\omega)$, placed at coordinates $\left(\vec{r}_{s}=0, z_{S}\right)$, can be represented as a sum over the vertical adiabatic modes.

In the particular statement of the problem outlined here, the index of refraction for horizontal rays depends only on $y$, reflecting the along-shore symmetry of the system. The time of propagation of a signal along a ray trajectory can be found as a curvilinear integral over the trajectory of a horizontal ray

$$
t_{l}(\omega, T)=\int_{R_{0 M}} \frac{d s}{v_{l}^{g r}(\omega)},
$$

where $v_{l}^{g r}(\omega)$ is the group velocity of mode $l$ traveling as a horizontal ray with initial and final coordinates at the source and receiver along path $R_{0 M}$. Calculations of the horizontal ray patterns that can explain the observed effects in accordance with the theory of horizontal rays are shown in Fig. 2. In this example, a simple solitary IW is approaching an acoustic track in the horizontal plane. The experimentally measured values of the IW parameters such as the amplitude $(20 \mathrm{~m})$, envelope and velocity of the NIW $(0.9 \mathrm{~m} / \mathrm{s})$,
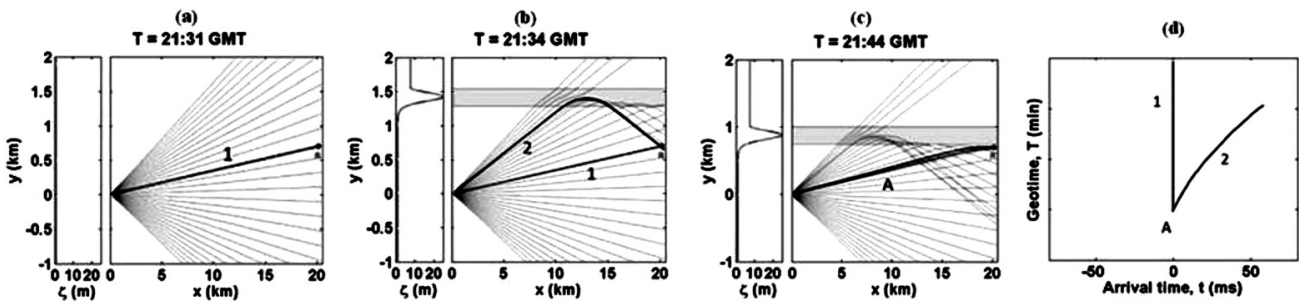

Fig. 2. Behavior of horizontal ray pattern for three positions of the NIW front as it approaches the acoustic track. (a) $T=21: 31$ GMT, when NIW is far from the track and only the primary (direct) ray path (labeled 1 on plot) is reaching the receiver, (b) $T=21: 34$ GMT, when NIW is in the vicinity of the acoustic track. We note the formation of the secondary horizontal (refracted) ray path (labeled 2 on plot) is reaching the receiver, (c) $T$ $=21: 44$ GMT, when NIW is arriving at acoustic track and both direct and refracted rays have reached the receiver, this situation is labeled A on the plot, (d) arrival time of the primary (1) and secondary (2) rays merging at point $\mathrm{A}$ as the NIW moves toward the acoustic track. This is calculated for mode 4 using Eq. (1). 
the sound speed profile it generates, and its width $(300 \mathrm{~m})$ and direction with respect to the acoustic track (taken from three horizontal positions with respect to the acoustic track) are utilized in this calculation. The NIW is moving along the $Y$-axis, having a plane wave front parallel to the $X$-axis (the calculations are carried out for mode 4).

In Fig. 2(a), the NIW is far from the acoustic track, so that we have only a direct path from source to the receiver $\mathrm{R}$ [such as the ray labeled "1" in Fig. 2(a)]. After some later time, for example, at $T=21: 34$ GMT, the NIW front is close enough to the acoustic track that some refracted rays reach the receiver. This case falls into the multipath regime, and we have two signals at the receiver R [Fig. 2(b)], corresponding to two horizontal rays, shown by thick curves labeled " 1 " and " 2 " on the plot. A few minutes later, at $T=21: 37$ GMT in Fig. 2(c), the location of the NIW is such that the receiver is now in the vicinity of a caustic, as the two rays reaching the receiver $\mathrm{R}$ are very close to each other and their arrival times are almost the same. This case is shown by two dark lines representing the direct and refracted paths ( 1 and 2) and it is labeled "A" on the plot. After this, the receiver falls into the neighboring shadow zone. Figure 2(d) shows the calculated modal arrival times as a function of geotime and is calculated using Eq. (1). In this figure, the arrival of the direct path continues for about $5 \mathrm{~min}$ as shown by the vertical line labeled "1" corresponding to Fig. 2(a). The refracted path that starts to arrive around 21:34 GMT [see Fig. 2(b)] moves along the curve labeled " 2 " corresponding to the travel time of rays in Fig. 2(b). This curved line mergers with the direct path as the IW gets closer to the track and finally meets it at point " $\mathrm{A}$ " when the two rays overlap corresponding to the rays shown in Fig. 2(c). It is seen that the ray arrival characteristics shown in Fig. 2(d), that are calculated for mode 4, are very similar to what is observed in the data (see Fig. 1, mode 4).

\section{Vertical mode and horizontal PE calculation}

A vertical mode and horizontal PE calculation is performed to obtain the intensity of modal arrivals in a simplified environmental model. The simplified environmental model uses sound speed profile measurements on the WHOI VLA and assumes straight wave front with an angle $4^{\circ}$ (counter clockwise) relative to the acoustic propagation path. The propagation speed of the modeled NIW packet is taken to be $0.8 \mathrm{~m} / \mathrm{s}$. Also, there is no variability along the modeled wave front. The water depth is $79.2 \mathrm{~m}$ (from experimental data at the VLA site) and assumed to be constant over the model domain, thus neglecting the real bathymetry variability. The range-independent bottom model is taken from the previous study by Jiang et al. ${ }^{6}$ A standard PE program (RAM PE) has been modified to solve the horizontal refraction equation, and a specific modification is made to change the preset point source self-starter to a line source self-starter for the two-dimensional horizontal refraction equation. In this simulation, a broadband signal that contains eight periods of a $300 \mathrm{~Hz}$ sine wave and tapered by a Hann window is transmitted from a fixed source (source-to-receiver distance is the same as the experiment). Note that $99.95 \%$ of the source energy is contained in the $150 \mathrm{~Hz}$ bandwidth centered at $300 \mathrm{~Hz}$. The source depth in the model is $69.2 \mathrm{~m}$, which has the same height from the bottom as the NRL300 source.

Figure 3 shows the modeled arrival of the first six modes versus geotime. The general arrival patterns agree with the data and modal ray theory quite well [see Figs. 1 and 2(d) for comparison]. If, for example, we look at mode 4, a secondary path initiates when the NIW approaches the acoustic track and merges with the primary path of this mode's arrival when the NIW arrives at the track. The total interval of geotime seen in the model is the same as the data, but the modeled geotime is delayed $1.5 \mathrm{~min}$ to compensate for the slightly curved front and NIW variability along the front observed in the data. The appearance and merging of the multipath versus geotime and the correspondence between the modeled and experimental results for the moving NIW is clearly shown. However, there are some second order effects missing in the 

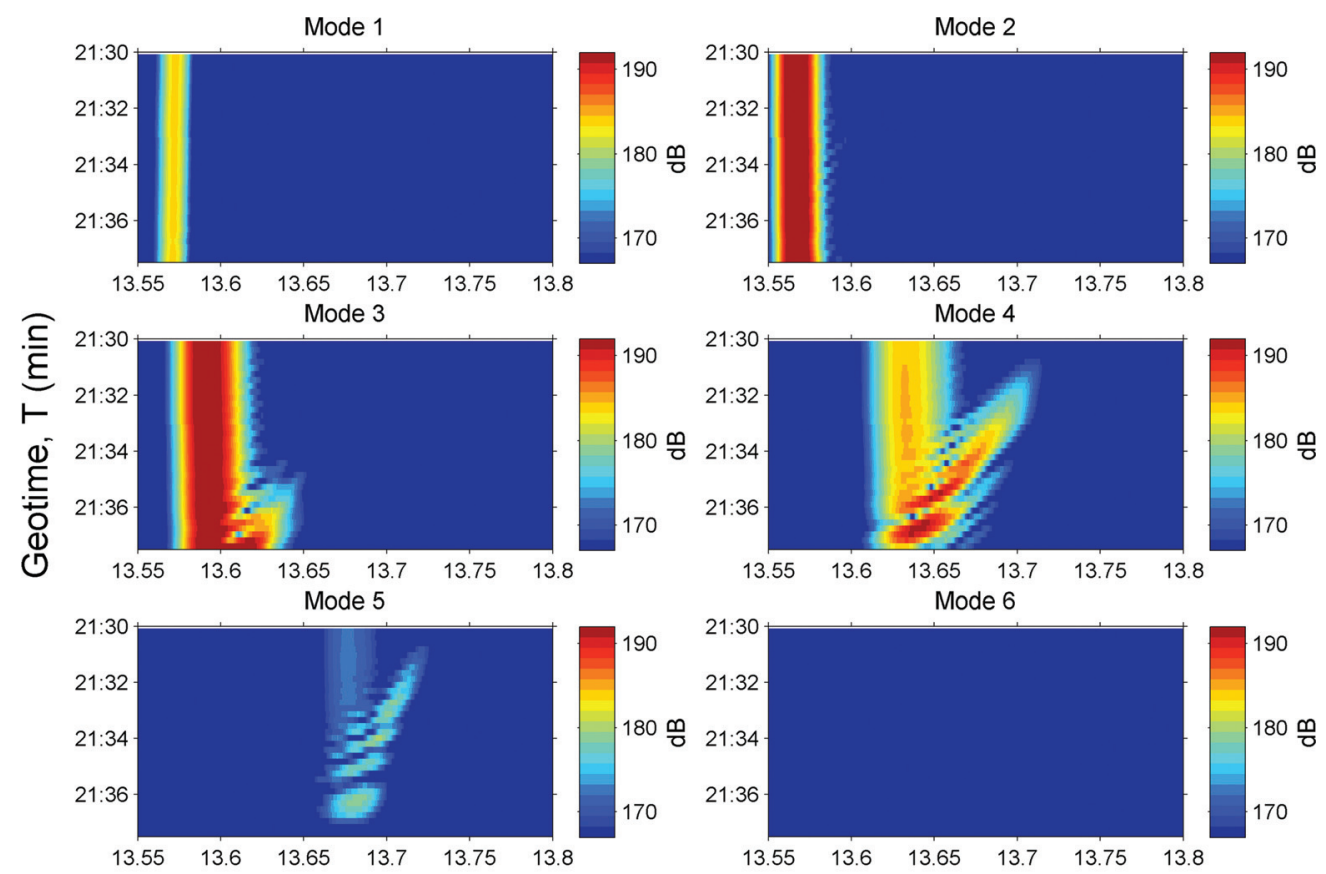

Travel time, $\mathrm{t}(\mathrm{sec})$

Fig. 3. Arrival of modes 1-6 calculated using vertical mode and horizontal PE from geotime $T=21: 30-21: 37$ GMT on August 17, 2006, corresponding to the data shown in Fig. 1. The center frequency is $300 \mathrm{~Hz}$ with 75 $\mathrm{Hz}, 3-\mathrm{dB}$ bandwidth.

model, such as the oscillations of the intensity fringes. The possibility, even at such low grazing angles, of modal energy transfer and coupling among different modes also needs to be investigated in the future. Comprehensive model/data comparison also requires reconstruction of fully $3 \mathrm{D}$ environmental parameters during this IW event, a complicated task that is beyond the scope of this express letter.

\section{Discussion}

In this section, we look in some further detail at the data features and how they correspond to the horizontal ray model, and the vertical mode and horizontal PE models, on a point-by-point basis. Typical values of the perturbation to the refraction index in the horizontal plane due to IW with amplitude $\sim 10-15 \mathrm{~m}$ are in the order of $|\mu| \sim 5 \times 10^{-3}-10^{-2}$. The difference in the arrival time for the horizontal rays propagating over the SW06 distances from the source to receiver (about $20 \mathrm{~km}$ in this paper) can thus be $\sim 0.1 \mathrm{~s}$. Given a rough speed of $0.7-1.5 \mathrm{~m} / \mathrm{s}$ for the solitons in SW06 means that the multipath interference pattern shown in the data could remain visible for about 10-20 min. This is consistent with the time duration of the "between wave ducting" effect that has been seen, ${ }^{4}$ as the average spacing between solitons is of the order of $1 \mathrm{~km}$.

At $T=21: 32$ GMT, the appearance of the secondary energy corresponds to a known position of the NIW since the ship's radar images and the thermistors data confirm the position. At this geotime, refracted horizontal rays initiate their arrival at the receiver as shown in Fig. 1 (e.g., see mode 4). The delay time of the refracted ray relative to the "direct path" signal (about $0.1 \mathrm{~s}$ in Fig. 1) corresponding to the difference in the two ray paths decreases during the following few minutes as the NIW front approaches the acoustic track. As the NIW gets close to the acoustic track, the secondary horizontal ray path appears and eventually overlaps with the primary path. The 
energy of the secondary arrival path can in general be a result of refraction or mode coupling. However, if the mode coupling is part of the contribution, it must be a very small part due to the small grazing angles involved. Details of mode coupling mechanism for a similar case is shown in Ref. 7 and falls in the category of higher order effects. Other observable effects, for example, temporal fluctuations of intensity such as the intensity fringes seen in the data, are also higher order effects that need to be considered in more detail.

This multipath effect reported here could not be caused by the signal processing because the matched filter output has a high signal to noise ratio, much greater than the side lobes (i.e., $>30 \mathrm{~dB}$ ). Therefore, the side lobes cannot be part of (or the cause of) the secondary arrival path that is seen in the arrival of the fourth mode. Here we note that modal cross talk has occured due to the array tilt, but it is also noted that the secondary arrival of mode 4 is seen in the pulse arrival time spread as depth versus geotime.

\section{Conclusion}

Three dimensional multipath pulse arrivals produced by the refraction of sound waves from an approaching NIW front have been experimentally observed. The incoming IW front acts as a moving frontal layer refracting sound in the horizontal plane. As the NIW front moves toward the acoustic track, a secondary (refracted) ray path in the horizontal plane approaches the primary (direct) ray path and finally merges into it as the NIW reaches the track. Received acoustic signals are filtered into acoustic normal mode arrivals using a vertical hydrophone line array. Using a simplified model of the NIW (i.e. straight IW front between acoustic source and receiver) with parameters taken from the field measurements, the theoretical and numerical results are in support of the proposed mechanism showing the arrival of direct path prior to the NIW approach as well as the arrival of the refracted path when NIW is present. The features shown in the modeled results closely match those observed in the data.

\section{References and links}

${ }^{1}$ J. F. Lynch, J. A. Colosi, G. G. Gawarkiewicz, T. F. Duda, A. D. Pierce, M. Badiey, B. G. Katsnelson, J. E. Miller, W. Siegmann, C.-S. Chiu, and A. Newhall, "Consideration of fine-scale coastal oceanography and 3-D acoustic effects for the ESME sound exposure model," IEEE J. Ocean. Eng. 31(1), 33-48 (2006).

${ }^{2}$ M. Badiey, B. Katsnelson, J. Lynch, and S. Pereselkov, "Frequency dependence and intensity fluctuations due to shallow water internal waves," J. Acoust. Soc. Am. 122(2), 747-760 (2007).

${ }^{3}$ M. Badiey, Y. Mu, J. F. Lynch, J. R. Apel, and S. N. Wolf, "Temporal and azimuthal dependence of sound propagation in shallow water with internal waves," IEEE J. Ocean. Eng. 27, 117-129 (2002).

${ }^{4}$ J. Luo, M. Badiey, E. A. Karjadi, B. Katsnelson, A. Tskhoidze, J. F. Lynch, and J. Moum, "Observation of sound focusing and defocusing due to propagating nonlinear internal waves," J. Acoust. Soc. Am. Express Letters 124(3), EL66-EL72 (2008).

${ }^{5}$ A. E. Newhall, T. F. Duda, K. von der Heydt, J. D. Irish, J. N. Kemp, S. A. Lerner, S. P. Liberatore, Y.-T. Lin, J. F. Lynch, A. R. Maffei, A. K. Morozov, A. Shmelev, C. J. Sellers, and W. E. Witzell," Acoustic and oceanographic observations and configuration information for the WHOI moorings from the SW06 experiment," Technical Report WHOI-2007-004, Woods Hole Oceanographic Institution, May 2007.

${ }^{6}$ Y.-M. Jiang, R. Chapman, and P. Gerstoff, "Short range travel time geoacoustic inversion with vertical array," J. Acoust. Soc. Am. Express Letters 124(3), EL135-EL140 (2008).

${ }^{7}$ B. Katsnelson, V. Grigorev, M. Badiey, and J. F. Lynch, "Temporal sound field fluctuations in the presence of internal solitary waves in shallow water," J. Acoust. Soc. Am. Express Letters 126(1), EL41-EL48 (2009). 